\title{
Neuroretinite unilateral subaguda difusa (DUSN): atualização continuada
}

\section{Diffuse unilateral subacute neuroretinitis (DUSN): current update}

\author{
Alexandre Antonio Marques Rosa ${ }^{1}$, Taurino dos Santos Rodrigues Neto ${ }^{2}$
}

\begin{abstract}
RESUMO
A neurorretinite subaguda difusa unilateral (DUSN) é uma forma de uveíte que pode potencialmente levar à cegueira. No Brasil e em outras partes da América do Sul, a neurorretinite subaguda difusa unilateral cada vez mais é considerada uma causa importante de uveíte posterior em crianças e em adultos jovens e saudáveis. Se diagnosticada e tratada ainda em fase inicial, permite uma resolução dos sintomas com melhora da acuidade visual. Caso progrida para a fase tardia, poderá acarretar uma perda visual significativa. Nesse estudo, por meio de uma revisão da literatura, descreve-se as principais características desta doença, incluindo os seguintes aspectos: histórico, etiologia, fisiopatologia, quadro clínico, diagnóstico, diagnóstico diferencial e tratamento.
\end{abstract}

Descritores: Retinite/fisiopatologia; Retina/lesões; Retinite/epidemiologia; Retinite/ diagnóstico; Infecções oculares parasitárias/etiologia; Neurite optica/patologia

\begin{abstract}
Diffuse unilateral subacute neuroretinitis (DUSN) is a form of uveitis that can potentially lead to blindness. In Brazil and other parts of South America, diffuse unilateral subacute neuroretinitis is an important cause of posterior uveitis in children and healthy young adults. If diagnosed and treated in early stage, allows a resolution of symptoms with improvement of visual acuity. If the disease progresses to the late stage, can result in significant visual loss. In this study, through a literature review, we describe the main characteristics of this disease, including the following aspects: history, etiology, physiopathology, clinical features, diagnosis, differential diagnosis and treatment.
\end{abstract}

Keywords: Retina/physiopathology; Retina/injuries; Retina/diagnosis; Retina/parasitology; Eye Infections, Parasitic/etiology; Optic neuritis/pathology

\section{INTRODUÇÃO}

A uveíte é uma doença inflamatória intraocular que depende de diversos fatores socioeconômicos, geográficos e culturais ${ }^{(1)}$. Sua ocorrência varia de acordo com os agentes causais mais prevalentes em cada região. Em muitos países, as infecções parasitárias são uma causa importante de uveíte ${ }^{(2)}$. A neurorretinite subaguda unilateral difusa (DUSN) pode, de forma secundária, levar a uma inflamação na úvea e potencialmente levar à cegueira(3).

\section{HISTÓRICO}

DUSN é uma doença infecciosa que foi descrita primeiramente por Gass e Scelfo, em 1978(4). Uma larva, cuja etiologia ainda não foi bem estabelecida, é responsável por promover um processo inflamatório e degenerativo na retina posterior. No Brasil, a doença foi descrita inicialmente em $1991^{(5)}$. Em 1992, foi realizado a primeira identificação da larva na retina ${ }^{(6)}$. Apesar de ser primariamente unilateral, Souza et al., (1999)(7) descreveu a primeira evidência de DUSN afetando os dois olhos.

\section{ETIOLOGIA}

Está associada com a infecção por nematódeos de diferentes tamanhos e espécies, no geral são descritos dois tamanhos de larvas, a menor medindo de 400 a $1.000 \mu \mathrm{m}$, predominando na América Latina e sul dos Estados Unidos( ${ }^{(8)}$ (Figura 1), e a maior medindo de 1.500 a $2.000 \mu \mathrm{m}$, que prevalece no norte e meio-oeste dos Estados Unidos $^{(9-11)}$. Em 1999, foi descrito o primeiro caso de DUSN no Bra- sil causado por um nematódeo medindo entre 1.500 a $2.000 \mu \mathrm{m}$, o qual foi identificado como provável Baylisascaris procyonis, que parasita o intestino de pequenos carnívoros, incluindo guaxinins e gambás ${ }^{(12)}$. Embora sua etiologia seja atribuída principalmente a espécies do filo Nematoda, existem relatos de DUSN ocasionada por uma larva com tamanho aproximado de 555 × $190 \mu \mathrm{m}$, cuja origem seja provavelmente a Alaria mesocercaria, um trematódeo, cuja provável fonte de infecção foi a ingestão de patas de rãs contaminadas e mal cozidas ${ }^{(13)}$.

A maioria dos estudos defendem que diferentes agentes possam ser consideradas como prováveis causadores da DUSN ${ }^{(8,14)}$. Os nematóides menores seriam provavelmente: Ancylostoma caninum, enquanto que as larvas maiores seriam do Baylisascaris procyonis $(3,8,15,16)$.

\section{FISIOPATOLOGIA}

A etiopatogenia da DUSN está relacionada à presença de uma larva no espaço sub-retiniano, a qual promoveria um processo degenerativo extenso na retina, com infiltração de células inflamatórias, com agregado de macrófagos e gliose podendo afetar todas as camadas, com perda parcial de células ganglionares, também ocorreria uma agressão tóxica às células bipolares, o que se justifica nas alterações observadas no eletrorretinograma ${ }^{(17)}$.

$\mathrm{Na}$ infecção ativa ou aguda, pode haver infiltração de eosinófilos associado a retinocoroidite, vitreíte e vasculite retiniana. A migração do verme pela retina, associado ao processo inflamatório levaria a uma degeneração do epitélio pigmentado, com descontinuidade na transmissão da informação entre as células retinianas ${ }^{(18)}$.
Submetido para publicação: 1 de março de 2012

Aceito para publicação: 2 de abril de 2013

Trabalho realizado na Universidade Federal do Pará - UFPA, Belém (PA) - Brasil.

${ }^{1}$ Médico, Departamento de Oftalmologia, Universidade Federal do Pará - UFPA, Belém (PA) - Brasil.

${ }^{2}$ Estudante de Medicina, Universidade do Estado do Pará - UFPA, Belém (PA) - Brasil.
Financiamento: Não houve financiamento para este trabalho.

Divulgação de potenciais conflitos de interesse: A.A.M.Rosa, Nenhum; T.S.Rodrigues Neto, Nenhum. Endereço para correspondência: Alexandre Antônio Marques Rosa. Av. Conselheiro Furtado, 2865 Sobreloja - Sala 4 - Belém (PA) - 66060-000 - Brasil -E-mail: alexandre_ros@hotmail.com 


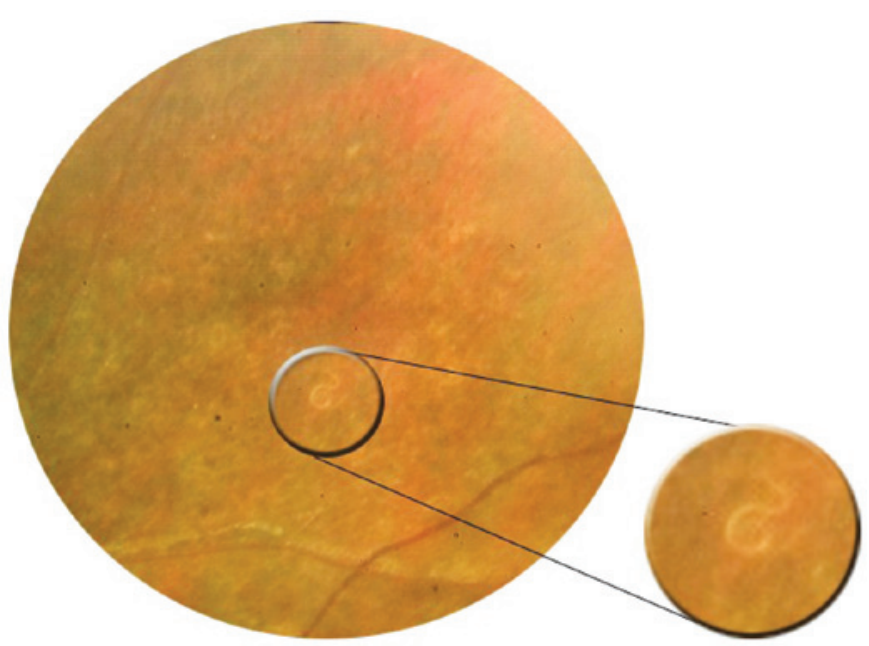

Figura 1. Retinografia demonstrando a larva sub-retiniana de menor tamanho que predomina na América Latina e sul dos Estados Unidos. (Cortesia Prof. Dr. Vitor Cortizo, Terezina - PI).

\section{QUADRO CLÍNICO}

Normalmente, a doença cursa sem manifestações clínicas ou laboratoriais que possam sugerir uma infecção parasitária sistêmica ao iniciar os sintomas oculares ${ }^{(19)}$. Atinge principalmente a faixa etária infantil e adultos jovens. O curso clínico da doença é caracterizado por uma inflamação intraocular que tende a ser unilateral e difusa ${ }^{(20)}$ muitas vezes com caráter progressivo, culminando com uma atrofia óptica irreversível|(18)

Na fase aguda da doença, geralmente há diminuição da acuidade visual, que pode estar relacionado a vitreíte, retinite e edema de disco óptico. Pode haver também a presença de escotomas central ou paracentral no campo visual(21). Observam-se lesões brancoacinzentadas na retina, que podem ser transitórias, difusas ou focais, e podem estar relacionadas a possível localização do nematódeo no espaço sub-retiniano (Figura 2A e 2B). Após uma semana, em geral, devido ao movimento da larva para outra área do olho, as lesões branco-acinzentadas ativas somem e podem reaparecer distante ou adjacente a área inicial da lesão(22). Achados menos frequentes incluem hemorragias retinianas, cisto macular - no qual se acredita que seja consequente a coalescência dos espaços císticos do edema macular cistoide ${ }^{(23)}$, exsudatos perivenosos, descolamento localizado da retina neurosensorial e iridociclite com hipópio, que pode estar relacionado com a morte do nematódeo durante o curso da doença $a^{(4,24)}$.

A identificação do verme sub-retiniano móvel é um achado patognomônico na DUSN. Apesar de poder acometer todas as camadas, sua localização é mais frequentemente encontrada nas camadas externas da retina. A larva apresenta coloração esbranquiçada, e ao exame de fundo de olho é identificada como uma linha tênue, afilada em ambas as extremidades e geralmente assume um formato de "S". $\mathrm{Na}$ fase tardia, pode se observar estreitamento arteriolar difuso na retina, palidez do disco óptico e degeneração do epitélio pigmentado $^{(4,18,21)}$ (Figura 3).

A identificação da larva pode ser realizada tanto nas fases aguda quanto na crônica. A presença da larva define o diagnóstico, entretanto a sua ausência não o exclui, uma vez o diagnóstico pode ser estabelecido com base nos achados clínico-oftalmológicos, configurando o quadro de DUSN presumida. Em um estudo com 121 pacientes, 7,43\% encontrava-se em fase aguda, os achados clínicos mais frequentes foram túneis retinianos $(91,7 \%)$, alterações focais do epitélio pigmentado da retina (89,3\%), pontos esbranquiçados $(80,2 \%)$ e atrofia do nervo óptico $(76,9 \%){ }^{(25)}$
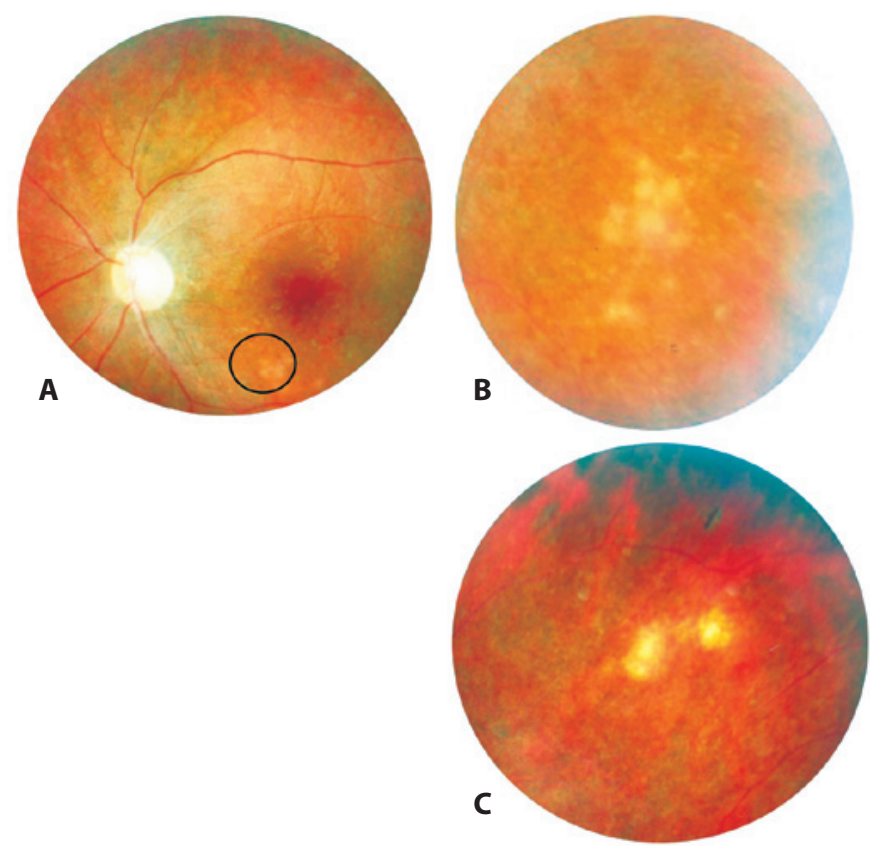

Figura 2. Retinografia de um paciente com diagnóstico de DUSN presumível em fase aguda, tratado com albendazol. A) Olho afetado na fase aguda com as lesões brancoacinzentadas circuladas. B) Detalhe ampliado das lesões branco-acinzentadas na fase aguda - antes do tratamento.

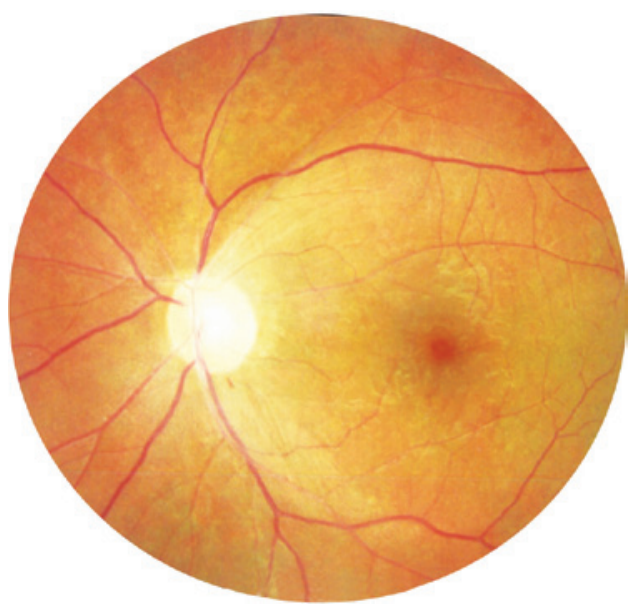

Figura 3. Retinografia de um paciente com diagnóstico de DUSN presumível em fase crônica, onde se observa palidez do disco óptico e estreitamento arteriolar.

\section{DIAGNÓSTICO}

O estabelecimento de um diagnóstico precoce na DUSN é de extrema importância, por ser uma das poucas causas preveníveis de cegueira na infância, a erradicação do verme nos estágios iniciais poderá evitar a progressão para perda visual(19).

O diagnóstico de certeza da DUSN se baseia no encontro e identificação do nematoide móvel sub-retiniano. Pacientes que apresentam apenas os sinais do estágio agudo, na ausência da larva, podem ter um diagnóstico de DUSN presumível. Além dos achados fundoscópicos, outros exames complementares podem auxiliar no diagnóstico e no acompanhamento da evolução da doença ${ }^{(26)}$.

A realização de teste sorológico nos pacientes portadores de DUSN é extremamente controversa. Em um estudo, em 1983, não 
foi encontrado sorologia positiva para T. $\operatorname{canis}^{(8)}$. Em 2000, foram confirmados 5 casos de neurorretinite por toxocaríase utilizando a sorologia(27). Entretanto, Casella $(2001)^{(28)}$ em 8 casos estudados, observou sorologia positiva em apenas 3. Gass, Braunstein (1983) não identificaram testes sorológicos positivos.

Os testes sorológicos apresentam um valor limitado no estabelecimento diagnóstico de DUSN, sendo mais indicados quando se quer excluir outras doenças como diagnóstico diferencial(22). Além disso, devido a possibilidade do compartilhamento de antígenos por diferentes nematódeos, a interpretação dos testes sorológicos, a fim de confirmar o agente etiológico, está sujeita a erros ${ }^{(18)}$.

Em 2002, foi realizado um estudo utilizando o eletrorretinograma multifocal como um teste sensível para localizar disfunções retinianas decorrentes da toxicidade local causadas pelos produtos metabólicos do verme, além de auxiliar na evolução após o tratamento do DUSN ${ }^{(29)}$.

O eletrorretinograma de campo total geralmente apresenta uma onda negativa como resposta aos estímulos em condições escotópicas, havendo uma redução seletiva na onda $b$, responsável pelo componente positivo, e um domínio da onda a negativa. Em geral, a onda $b$ é mais atingida que a onda $a, c o m$ a relação $b / a<1$. A presença constante de ERGs negativos pode indicar também que a doença, através de mecanismos que são discutidos, atinge sistematicamente as células bipolares ${ }^{(30-33)}$. Apesar da redução das respostas, Audo et al., (2006) ${ }^{(33)}$ defende que, no olho afetado, nenhum ERG apresenta resposta nula, além disso, o olho saudável sempre possui um ERG normal. O comprometimento das respostas no exame acompanha a progressão da doença, em que cada vez mais fotorreceptores são afetados, podendo ocorrer também uma disfunção do nervo óptico, provavelmente anterior ao envolvimento da retina, o que contribui para a redução da acuidade visual.

A angiofluoresceinografia, nos estágios iniciais, permite mostrar uma hipofluorescência na área em que se encontra as lesões brancoacinzentadas. Além disso, extravasamento de corante a partir dos capilares do disco óptico também pode ser observado. Em estágios mais avançados, a angiografia pode evidenciar a perda do epitélio pigmentado da retina, que se manifesta ao exame como hiperfluorescência transmitida ${ }^{(34)}$.

A tomografia de coerência óptica (OCT) é um método não invasivo que auxilia no diagnostico e no acompanhamento da doença, uma vez que permite a mensuração da espessura da camada de fibras nervosas da retina (CFNR) ${ }^{(35)}$. Em uma pesquisa utilizando OCT em 38 pacientes, 53\% apresentaram a larva viva localizada, o autor não observou diferença significante da espessura da CFNR entre os pacientes com diagnóstico de DUSN presumida e aqueles em que o verme foi identificado, porém encontrou-se uma relação entre a piora da acuidade visual e a diminuição da espessura ${ }^{(29)}$. Quando comparado ao olho saudável, foi demonstrado uma diferença significante entre as espessuras da CFNR(32). O principal achado observado na OCT consiste na atrofia da camada de fibras nervosas retinianas, outros achados menos usuais observados neste exame são: (1) edema localizado nas áreas afetadas pela larva, podendo ocorrer a formação de uma membrana epirretiniana foveal, presente na fase aguda e que pode regredir com o tratamento ${ }^{(35)}$, e (2) presença de cistos maculares ${ }^{(36)}$. Milan Shah et al., (2011) ${ }^{(37)}$ reportou 3 casos de DUSN com achados no OCT de domínio espectral (SD-OCT) de neovascularização retiniana, que foi precedida por uma atrofia progressiva do epitélio pigmentado.

Os achados de OCT são importantes para diferenciar de maneira significativa a DUSN da retinite punteada externa, causada pelo Toxoplasma gondii, a qual não apresenta atrofia de camadas de fibras nervosas ${ }^{(38)}$. Este tipo de atrofia está presente mesmo em casos em que a acuidade visual ficou relativamente preservada ${ }^{(35)}$.

\section{DIAGNÓSTICO DIFERENCIAL}

A DUSN é uma doença que pode simular várias outras desordens. O diagnóstico diferencial pode ser feito de acordo com o estágio em que ela se apresenta.
Na fase aguda, há a presença de lesões branco-acinzentadas e o diagnóstico diferencial pode ser feito com as outras doenças que compõem a síndrome dos pontos brancos (white dot syndrome) um grupo de condições inflamatórias idiopáticas que pode envolver tanto a retina quanto a coroide - nela inclui-se a epiteliopatia pigmentar placoide multifocal aguda posterior, coroidite serpenginosa, coroidite multifocal, panuveíte, retinocoroidopatia de Birdshot, retinite punteada externa e histoplasmose. Outras doenças que também podem fazer diagnóstico diferencial nesta fase são toxoplasmose, retinite herpética multifocal, citomegalovírus, abscessos bacterianos ou fúngicos e doença de Behçet. Quando presente a perivasculite, a sarcoidose pode entrar como diferencial. Em estágio precoce, na vigência de edema do disco óptico, a neurite retrobulbar idiopática, neuroretinite pós-vírus e o papiledema podem ser um possível diagnóstico ${ }^{(7,18,35,39)}$.

Na fase crônica, na vigência da atrofia do epitélio pigmentado da retina, deve-se considerar também a retinite pigmentosa unilateral, a corioretinopatia secundária ao trauma e a atrofia coriorretiniana por oclusão da artéria oftálmica. Nos casos de atrofia óptica, deve-se investigar também neurite óptica, lesões compressivas e neuropatia óptica isquêmica(7,18,19,26).

\section{TRATAMENTO}

Classicamente, a fotocoagulação a laser é o único tratamento recomendado para DUSN, uma vez que a larva seja localizada, porém a sua identificação ao exame oftalmológico é extremamente difícil, por ser uma tarefa desgastante e que demanda um pouco de tempo, porém com um exame minucioso seria possível a sua identificação em alguns pacientes ${ }^{(40)}$.

Caso o diagnóstico seja feito em fase inicial, seguido pela rápida localização e destruição da larva pela fotocoagulação a laser, é possível promover uma melhora na acuidade visual dos pacientes ${ }^{(8)}$.

A destruição da larva por fotocoagulação em olhos na fase crônica, não melhora a acuidade visual. Garcia et al., (2004)(41) estudou 22 olhos com DUSN na fase tardia, com a identificação da larva, após o tratamento com laser apenas dois olhos apresentaram melhora na acuidade visual. Apesar do tratamento na fase tardia não melhorar significativamente a acuidade visual, com ele é possível impedir a progressão da doença e promover uma melhora do campo visual ${ }^{(40)}$.

A fotocoagulação tem mostrado bons resultados quando realizada nas fases iniciais da doença e pode ser indicada quando o nematódeo encontra-se afastado da mácula. Quando a larva estiver sobre a região macular, o uso do laser poderia promover lesão adicional no local ${ }^{(42)}$. Neste caso ou quando o diagnóstico é feito na ausência da visualização do verme, o tratamento farmacológico pode ser uma alternativa segura e eficaz ${ }^{(43)}$.

Em 1992, foi publicado o primeiro caso de tratamento oral para DUSN presumível, utilizando o tiabendazol, o qual se mostrou eficaz, principalmente em pacientes com inflamação vítrea moderada a severa ${ }^{(44)}$. O tiabendazol e ivermectina tem sido utilizados em casos de DUSN presumida, sendo a última, uma alternativa nos casos de ineficácia ou intolerância ao tiabendazol ${ }^{(44,45)}$.

Em casos de DUSN confirmada, a uso do tiabendazol e ivermectina não mostrou efeito, necessitando de fotocoagulação para destruição da larva ${ }^{(46)}$. A terapia farmacológica com tiabendazol e ivermectina parece não ser eficaz na destruição do organismo em todos os pacientes, especialmente naqueles com inflamação vítrea mínima, onde há baixa penetração ocular do medicamento(22,26). Além disso, o sucesso do tratamento com estas drogas ocorreu em casos de diagnóstico presumível, onde a comprovação do efeito terapêutico é difícil, o que também poderia explicar o insucesso no tratamento registrado por alguns autores ${ }^{(46,47)}$. Independente da droga ou esquema utilizado, a terapia farmacológica tem revelado bons resultados. Recentemente, o uso de albendazol tem assumido um papel de importância no tratamento de DUSN presumida, mostrando 
ser uma alternativa segura e eficaz, além de possuir uma capacidade maior de cruzar a barreira hematorretiniana ${ }^{(40,46,48)}$. A dose e a duração do tratamento para a DUSN ainda não são bem esclarecidas. Muitos trabalhos têm utilizado com sucesso 400 mg diários, por 30 dias, com base no tratamento da neurocisticercose com albendazol, uma vez que as duas doenças apresentam alguns aspectos na patogênese e na clínica em comum ${ }^{(49)}$. Naqueles casos confirmados, vários estudos demonstraram eficácia deste esquema terapêutico ${ }^{(40,46,48)}$.

Outro esquema de tratamento utilizado na Venezuela foi de $200 \mathrm{mg}$ três vezes ao dia, por dez dias(50), baseado em um estudo experimental que visava manter constante a concentração plasmática da droga, sem que ultrapassasse seu limiar de toxicidade ${ }^{(51)}$.

Em outra pesquisa ${ }^{(52)}$, foi utilizado $400 \mathrm{mg}$ de albendazol, duas vezes ao dia, por dez dias, baseando-se no tratamento utilizado em infecções por Toxocara ${ }^{(53)}$, o qual é um possível agente etiológico de prevalência importante no Brasil. Observaram melhora significativa da visão em cinco dos seis pacientes com diagnóstico de DUSN presumida, além de melhora em alguns parâmetros do ERG multifocal.

Alguns autores optaram por um esquema combinado de tratamento com corticoides orais, associado a fotocoagulação e anti-helmíntico, porém não existe consenso para esta terapia combinada ${ }^{(54)}$. Além disso, o uso de corticoide apesar de ter demonstrado melhora nos casos que apresentam grande inflamação (vitreíte intensa ou reação de câmara anterior), seu uso em longo prazo não tem sido favorável, sendo contraindicado na grande maioria dos $\operatorname{casos}^{(55)}$.

\section{CONCLUSÃO}

A DUSN é uma importante causa de uveíte que, se diagnosticada e tratada ainda em fase inicial, permite uma resolução dos sintomas com melhora da acuidade visual. Caso progrida para a fase tardia, poderá acarretar em uma perda visual significativa.

A identificação da larva é um achado patognomônico, porém na ausência do nematódeo o diagnóstico pode ser presumido com base nos achados dos exames de fundo de olho.

O tratamento de escolha é a fotocoagulação, entretanto o seu uso é indicado apenas nos casos em que a larva for identificada. Em casos de diagnóstico presumível, o tratamento farmacológico pode ser uma alternativa segura e eficaz.

\section{REFERÊNCIAS}

1. Cimino L, Aldigeri R, Salvarani C, Zotti CA, Boiardi L, Parmeggiani M, et al. The causes of uveitis in a referral centre of Northern Italy. Internat Ophthalmol. 2010;30(5): 521-9.

2. Rathinam SR, Cunningham ET Jr. Infectious causes of uveitis in the developing world. Int Ophthalmol Clin. 2000;40(2):137-52.

3. Sabrosa NA. DUSN: a potentially blinding parasitic infection. Review Ophthalmol. 2010; 17(2):58-62.

4. Gass JD, Scelfo R. Diffuse unilateral subacute neuroretinitis. J R Soc Med. 1978;71(2): 95-111.

5. Oliveira AA, Oréfice F. Estudo de sete casos de neuroretinite difusa subaguda unilateral. Rev Bras Oftalmol. 1991;51(1):51-5.

6. Souza EC, Cunha SL. Neurorretinite sub-aguda unilateral difusa no Brasil: encontro da larva sub-retiniana. Arq Bras Oftalmol. 1992;55(6): 251-4.

7. Souza EC, Abujamra S, Nakashima Y, Gass DJ. Diffuse bilateral subacute neurorretinitis: first patient with documented nematodes in both eyes. Arch Ophthalmol. 1999. 117(10):1349-51

8. Gass JD, Braunstein RA. Further observations concerning the diffuse unilateral subacute neuroretinitis syndrome. Arch Ophthalmol. 1983;101(11):1689-97.

9. Goldberg MA, Kazacos KR, Boyce WM, Ai E, Katz B. Diffuse unilateral subacute neuroretinitis. Morphometric, serologic, and epidemiologic support for Baylisascaris as a causative agent. Ophthalmology. 1993;100(11):1695-701.

10. Kazacos KR, Raymond LA, Kazacos EA, Vestre WA. The raccoon ascarid: A probable cause of human ocular larva migrans. Ophthalmology. 1985;92(12):1735-44.

11. Sabrosa NA, Cunningham ET Jr, Arevalo JF. Ocular nematode and trematode infections in the developing world. Int Ophthalmol Clin. 2010;50(2):71-85.

12. Cialdini AP, Souza EC, Avila MP. The first South American case of diffuse unilateral subacute neuroretinitis caused by a large nematode. Arch Ophthalmol. 1999;117(10): 1431-2. Comment in: Arch Ophthalmol. 2000;118(11):1593.
13. McDonald HR, Kazacos KR, Schatz H, Johnson RN. Two cases of intraocular infection with Alaria mesocercaria (Trematoda). Am J Ophthalmol. 1994;117(4):447-55. Erratum in: Am J Ophthalmol. 1994;118(1):129.

14. Kazacos K, Vestre WA, Kazacos EA, Raymond LA. Diffuse unilateral subacute neuroretinitis: probable cause. Arch Ophthalmol. 1984;102(7):967-8.

15. Gass JD, Gilbert WR Jr, Guerry RK, Scelfo R. Diffuse unilateral subacute neuroretinitis.1978. Ophthalmology. 2003;23(6 Suppl):521-45

16. Dhir L, O' Dempsey T, Watts M. Cutaneous larva migrans with optic disc edema: a case report. J Med Case Rep. 2010;4(1):209.

17. Oréfice F, Bonfioli AA, Paranhos FRL. Neuroretinite subaguda unilateral difusa. In: Oréfice F, editor. Uveíte: clínica e cirúrgica. Rio de Janeiro: Cultura Médica; 1998. p.733-56.

18. Gass JD, Olsen KR. Diffuse unilateral subacute neuroretinitis. In: Ryan SJ, editor. Retina. $3^{\text {th }}$ ed. St Louis: CV Mosby; 2001. p.1669-78.

19. Abdalla M, Tannous M, Nunes HL, Aquino MV, Madeira D. DUSN: a importância do diagnóstico e do tratamento precoce. Apresentação de caso e revisão bibliográfica. Rev Revicience [Internet]. 2005[citado 2012 Nov 21];7(7):16-9. Disponível em: http:// www.cemahospital.com.br/images/revistarevicience/revista-reviencie-07.pdf

20. Quillen DA, Davis JB, Gottlieb JL, Blodi BA, Callanan DG, Chang TS, et al. The white dot syndromes. Am J Ophthalmol. 2004;137(3):538-50. Comment in: Am J Ophthalmol. 2004;138(4):686; author reply 686-7.

21. Gass JD. Diffuse unilateral subacute neuroretinitis. In: Gass JD, editor. Stereoscopic atlas of macular diseases: diagnosis and treatment. $4^{\text {th }}$ ed. St Louis: CV Mosby; 1997. p.622-8.

22. Kooragayala LM. Diffuse unilateral subacute neuroretinitis: differential diagnoses \& workup. New York; Medscape reference; 2011 [cited 2012 Aug 21]. Available from: http://emedicine.medscape.com/article/1226931-treatment

23. Vedantham V, Vats MM, Kakade SJ, Ramasamy K. Diffuse unilateral subacute neuroretinitis with unusual findings. Am J Ophthalmol. 2006;142(5):880-3.

24. Muccioli C, Belfort Jr R. Hypopyon in a patient with presumptive diffuse unilateral subacute neuroretinitis. Ocul Immunol Inflamm. 2000;8(2):119-21.

25. Garcia Filho CA, Gomes AH, Soares AC, Garcia CA. Clinical features of 121 patients with diffuse unilateral subacute neuroretinitis. Am J Ophthalmol. 2012;153(4):743-9.

26. Matos MA, Silva DD, Trigueiro SA, Arcoverde AL, Dias S, Kattah MZ. Relato de uma série de 30 casos presumíveis de neurorretinite subaguda unilateral difusa (DUSN). Arq Bras Oftalmol. 2001:64(2):103-7.

27. Park SP, Park I, Park HY, Lee SU, Huh S, Magnaval JF. Five cases of ocular toxocariasis confirmed by serology. Korean J Parasitol. 2000;38(4):267-73.

28. Casella AM, Machado RA, Tsuro A, Hato M, Costa R, Eid Farah M. Seria o Ancylostoma caninum um dos agentes da neurorretinite sub-aguda difusa unilateral (D.U.S.N.) no Brasil? Arq Bras Oftalmol. 2001;64(5):473-6.

29. Martidis A, Greenberg PB, Rogers AH, Velázques-Estades $L$, Baumal CR. Multifocal electroretinography response after laser photocoagulation of a subretinal nematode. Am J Ophthalmol. 2002;133(3):417-9.

30. Paranhos FR, Ávila MP, Paranhos A, Cialdini AP. Estudo estatístico de valores normais do eletrorretinograma: contribuição à padronização do exame. Arq Bras Oftalmol. 1997:60(3):278-84

31. Garcia CA, Oliveira AG, Lima CE, Rocha FN, Garcia Filho CA. Retinal nerve fiber layer analysis using GDx in 49 patients with chronic phase DUSN. Arq Bras Oftalmol. 2006; 69(5):631-5.

32. Vezzola D, Kisma N, Robson AG, Holder GE, Pavesio C. Morphologic and functional retinal changes in eyes with DUSN. Poster session presented at: ARVO 2011 Visionary genoma; 2011 May 1-5. Fort Lauderdale (EUA).

33. Audo I, Webster AR, Bird AC, Holder GE, Kidd MN. Progressive retinal dysfunction in diffuse unilateral subacute neuroretinitis. Br J Ophthalmol. 2006;90(6):793-4.

34. Veiga-Reis F, Oréfice $J$, Nehemy MB, Oréfice F. Neurorretinite sub-aguda unilateral difusa: videoangiografia digital com indicianina verde e eletrorretinograma. Rev Bras Oftalmol. 1999;58(7):525-33.

35. Casella AM, Farah ME, Souza EC, Belfort Jr R, Oguido AP. Retinal nerve fiber layer atrophy as relevant feature for diffuse unilateral subacute neuroretinitis (DUSN): case series. Arq Bras Oftalmol. 2010:73(2):182-5.

36. Vedantham V, Vats MM, Kakade SJ, Ramasamy K. Diffuse unilateral subacute neuroretinitis with unusual findings. Am J Ophthalmol. 2006;142(5):880-3.

37. Milan Shah DJ, Audina MB. Spectral domain OCT findings in Diffuse Acute Unilateral Neuroretinitis (DUSN) with subretinal neovascularization. Poster session presented at: ARVO 2011 Visionary genoma; 2011 May 1-5. Fort Lauderdale (EUA).

38. Souza EC, Casella AM. Clinical and tomographic features of macular punctate outer retinal toxoplasmosis. Arch Ophthalmol. 2009;127(10):1390-4.

39. Abu-Yaghi NE, Hartono SP, Hodge DO, Pulido JS, Bakri SJ. White Dot Syndromes: a 20-year study of incidence, clinical features, and outcomes. Ocul Immunol Inflamm. 2011;19(6):426-30.

40. Souza EC, Casella AM, Nakashima Y, Monteiro ML. Clinical features and outcomes of patients with diffuse unilateral subacute neuroretinitis treated with oral albendazole. Am J Ophthalmol. 2005;140(3):437-45. Erratum in: Am J Ophthalmol. 2006;141(4): $795-6$.

41. Garcia CA, Gomes AH, Garcia Filho CA, Vianna RN. Early-stage diffuse unilateral subacute neuroretinitis: improvement of vision after photocoagulation of the worm. Eye (Lond). 2004;18(6):624-7.

42. Natesh S, Nair KH, Nair K. Subretinal worm and repeat laser photocoagulation. Middle East Afr J Ophthalmol. 2010;17(2):183-5. 
43. Oueghlani E, O'Sullivan E, Pavesio CE. Diffuse unilateral subacute neuroretinitis in the United Kingdom. Int Ophthalmol. 2010;30(5):615-9.

44. Gass JD, Callanan DG, Bowman CB. Oral therapy in diffuse unilateral subacute neuroretinitis. Arch Ophthalmol. 1992;110(5):675-80.

45. Callanan DG, Davis JL, Cohen SM, Halperin L, Gass JD. The use of ivermectin in diffuse unilateral subacute neuroretinitis. Ophthalmology. 1993;100(9 Suppl):114-5.

46. Casella AM, Farah ME, Belfort Jr R. Antihelminthic drugs in diffuse unilateral subacute neuroretinitis. Am J Ophthal. 1998;125(1):109-11.

47. Oréfice F, Gonçalves ER, Siqueira RC, Nehemy MB. Estudo de 21 casos de neurorretinite subaguda unilateral difusa (DUSN); dois casos com larva móvel sub-retiniana. Rev Bras Oftalmol. 1994;53(6):23-45.

48. Malaguido MR, Casella AM, Malaguido DR. Tratamento clínico da neurorretinite subaguda difusa unilateral com albendazol. Arq Bras Oftalmol. 2007;70(5):814-22.

49. Singhi P, Dayal D, Khandelwal N. One week versus four weeks of albendazole therapy for neurocysticercosis in children: a randomized, placebo controlled double blind trial. Pediatr Infect Dis J. 2003;22(3):268-72.
50. Cortez R, Denny JP, Muci-Mendoza R, Ramirez G, Fuenmayor D, Jaffe GJ. Diffuse unilateral subacute neuroretinitis in Venezuela. Ophthalmology. 2005;112(12):2110-4

51. Delgado O, Botto C, Mattei R, Escalante A. Effect of albendazole in experimental toxocariasis of mice. Ann Trop Med Parasitol. 1989;83(6):621-4.

52. Rodrigues Neto TS, Frota de Almeida IN, Frota de Almeida LN, Frota de Almeida Sobrinho E, Gomes BD, Souza GS, et al. Retinal evaluation of patients with diffuse unilateral subacute neuroretinitis before and after albendazole therapy: electrophysiology and optical coherence tomography findings. Poster session presented at: ARVO $2011 \mathrm{Vi}$ sionary genoma; 2011 May 1-5. Fort Lauderdale (EUA).

53. Huapaya PH, Espinoza Y, Roldán W, Jiménez S. Toxocariosis humana: ¿problema de salud pública? An Fac Med. 2009;70(4):283-90.

54. Myint K, Sahay R, Mon S, Saravanan VR, Narendran V, Dhillon B. "Worm in the eye": the rationale for treatment of DUSN in south India. Br J Ophthalmol. 2006;90(9): 1125-7.

55. Reynalds JD, Olitsky SE. Pediatric uveitis. In: Hood C, Lowder CY. Pediatric retina. Berlin: Springer; 2011. p.452. 